\title{
Health implications of dietary habits in transition countries-a life course perspective
}

\author{
Carlo Agostoni ${ }^{1}$, Marco Silano ${ }^{2}$ and Giovanni Fattore ${ }^{3}$
}

n the present issue of Pediatric Research, Khadilkar et al. (1) have investigated, through a cross-sectional study, the interrelationships of dietary composition and physical activity with growth and body composition of roughly 5,000 urban Indian children, aged 3-18 years, from the urban upper socioeconomic class of India.

This study has shown that the mean daily energy and protein intakes as recommended dietary allowance were significantly lower in both boys and girls above 6 years, with $55 \%$ reduction in micronutrient intakes in older children. Of particular interest, it is a fact that the results of this study were adjusted also for mid-parental height $z$ score.

Multiple regression analysis showed a positive association of children height for age $Z$ scores (HAZ) with the mid parental height $z$ scores and protein density and a negative association with physical inactivity. Total dietary energy and fat intakes as well as percent energy from fat or carbohydrates were not related to the body fat or muscle mass percentage. Reduced nutrient intakes with a high fat intake contribute to a higher body fat percentage coupled with suboptimal growth. Consistently, adequacy of protein and antioxidant intakes and increasing moderate physical activity have been identified as essential factors for optimal growth and body composition in Indian children.

Within the limits of any large cohort trial, the main message for the nutritionists from India, a major "transition" country, is that body composition depends more on the type and quality of physical activity, than on the diet.

As a further relevant factor, the finding that mid parental height was a significant predictor of the children HAZ underlines the contribution of genetic factors toward final height either in isolation or by modulating nutrient intake and onset of puberty.

However, even if specific description of the individual dietary patterns (made up by well-characterized food classes) is not reported in the present paper, an emerging scenario of consumption of foods rich in carbohydrate and fats, but poor in proteins and micronutrients, is foreseen. Generally, this model includes few raw foods and higher amounts of processed food preparations, including the "so-called" junk foods.

Although social and economic changes are reported to rapidly affect both nutritional patterns and lifestyles in the low-income and socially disadvantaged classes in the developing and transition countries (2), we have evidences here that also within the upper socioeconomic classes of a country in rapid transition toward a "Westernized" form of economy, such as India, some dietary unbalances are taking place.

Disadvantaged people are more prone to the health consequences connected with a frequent consumption of qualitatively poor foods (also collectively known as "junk foods"). According to the World Health Organization, the social disadvantage is defined with regard to (a) socioeconomic aspects, including income, employment, education, and socioeconomic status, (b) sociocultural aspects, such as gender, ethnicity, religion, culture, and migrant status, (c) sociogeographical aspects, such as living in a deprived neighborhood or urban vs. rural area, and (d) age.

The question of young people coming from the upper socioeconomic classes and following unbalanced dietary habits in early life is worrying, since it seems to indicate a generational trend, difficult to be reversed in transition and developing countries. Nevertheless, some paradoxical situations should be considered in these settings. For instance, although in Western countries poor women are less prone to breastfeed their infants, in developing countries breastfeeding practice is less popular amongst rich people, since they consider this practice as a sort of limitation to the availability of their time and personal resources.

The association of qualitatively poor foods with unfavorable long-term outcomes should be considered through different stages in the life course, along with the actions to prevent their negative effects in a broader perspective, inclusive of their correlates. Epigenetic mechanisms are emerging as mediators linking early environmental exposures during pregnancy with programmed changes in gene expression that can alter offspring growth and development. Since epigenetic modifications may be reversible by definition, understanding

\footnotetext{
${ }^{1}$ Department of Clinical Sciences and Community Health, University of Milan, IRCCS Ospedale Maggiore Policlinico, Milan, Italy; ${ }^{2}$ Unit of Human Nutrition and Health, Department of Food Safety, Nutrition and Veterinary Public Health, Istituto Superiore di Sanità, Rome, Italy; ${ }^{3}$ Department of Policy Analysis and Public Management \& Centre for Research on Health and Social Care Management, Università Bocconi, Milan, Italy. Correspondence: Carlo Agostoni (carlo.agostoni@unimi.it) 


\section{Commentary | Commentary}

how epigenetic mechanisms contribute to the transgenerational transmission of metabolic dysfunctions may be crucial for the development of novel prevention strategies. Considering clinical and epidemiological data, it appears that interventions need to be initiated before conception, and therefore extended starting from adolescence, in males and, above all, females.

The term "metabolic rehabilitation" has been used by Ethan Sims to describe the importance of preconceptional metabolic control in order to avoid later metabolic dysfunction in the mother and her offspring (3). Therefore, any lifestyle (inclusive of dietary) intervention before conception becomes a viable paradigm to improve perinatal metabolic outcomes. Within this context, a socioeconomical intervention to support women when adolescent and through all their childbearing age is a preventive strategy, able to counteract the negative transgenerational metabolic unbalances that predispose to the non-communicable disorders (Figure 1). The multiplicative effects of such a type of global intervention compared with the punctiform effects expected from single actions against single foods or even individual dietary patterns are easily understandable, since also additive educational benefits should be expected from mothers to their offspring.

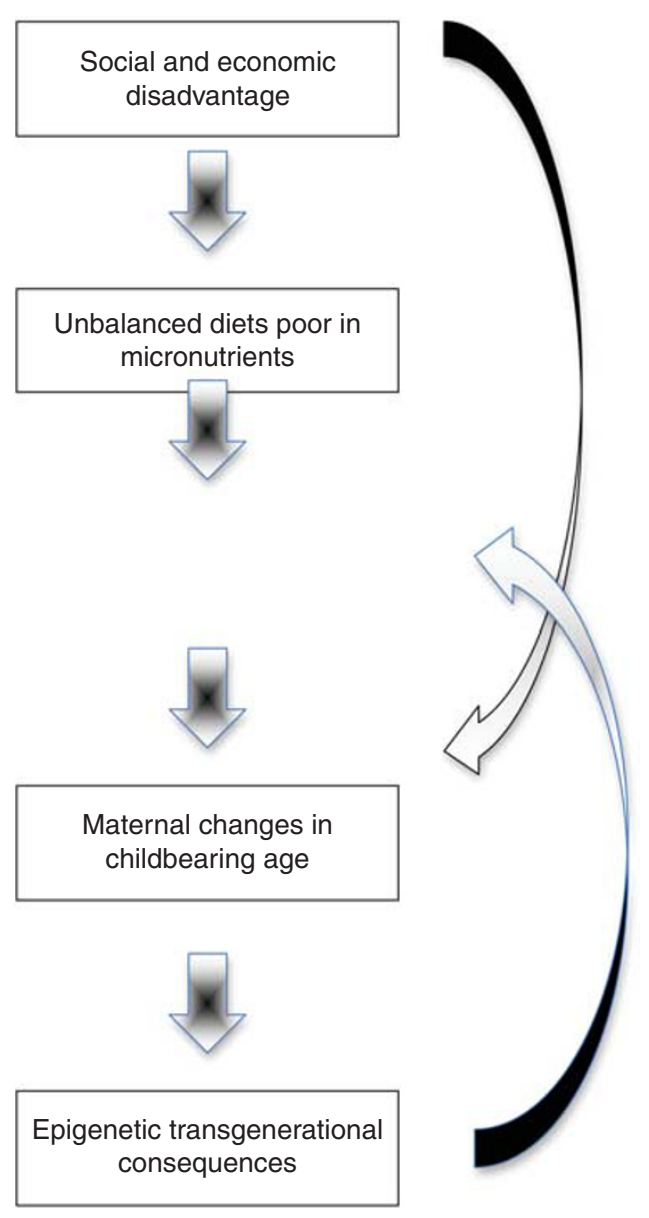

Figure 1. The vicious circle triggered by the social and economic disadvantage leading to an epigenetic transgenerational effect.
It is therefore clear that environmental conditions represent the link between genetic expression and disease. Epigenetic mechanisms are emerging as mediators linking early environmental exposures during fetal life with impaired growth and development. Epigenetic alterations encompass DNA methylation, histone modifications, chromatin remodeling, and microRNA synthesis. These mechanisms may activate, for instance, the genes controlling lipogenesis and low-grade inflammation in early pregnancy. These metabolic alterations occur before any changes in maternal phenotype. Therefore, trials of lifestyle interventions before pregnancy are needed to demonstrate the safety and efficacy for both the mother and her offspring, as already anticipated for childbearing age and conception.

If the consumption of unbalanced foods may be extrapolated as indicator of propensity to future chronic degenerative disorders in developing countries, within specific biologic and socioeconomic contexts, its intergenerational effect might be also mediated by its influence on food preferences and choice. A child used to industry-based food, instead of raw and whole, brings consequences on dietary pattern throughout her/his lifespan. In addition, this dietary choice is influenced by her/his economic condition that rarely improves from one generation to the following, especially in periods of economic constraints, and some experiments give biological plausibility to an intergenerational inherence of dietary habits associated with alteration of maternal stress hormone axis (4).

\section{Conclusions}

Any intervention aimed at reducing the impact of unbalanced diets (particularly if poor in micronutrients) should be addressed not only toward the individuals, but also in a life course prospective, at least to the prior generation, represented by young adolescent women (and men too, probably) at the beginning of their childbearing age. In addition, junk food consumption should be considered within a multifactorial framework, along with physical inactivity, and also unhealthy habits such as smoking and alcohol consumption. Interventions must extend beyond individual risk factors, involving the whole social texture in transition and developing countries, while favoring the integration and the promotion of immigrants within Western Countries, and continue throughout the individual life course. In agreement with Ioannidis "We should continue to explore other aspects of food and nutrition-such as food security, sustainability, social inequalities, famine, and impact of food production on climate change-that may also affect human societies and wellbeing through multiple pathways." (5)

\section{STATEMENT OF FINANCIAL SUPPORT \\ Intramural funding.}

Disclosure: The authors declare no conflict of interest.

\section{REFERENCES}

1. Khadilkar AV, Chiplonkar SA, Kajale NA, et al. Impact of dietary nutrient intake and physical activity on body composition and growth in Indian children. Pediatr Res 2017 (this issue). 


\section{Commentary | Agostoni et al.}

2. Darnton-Hill I, Nishida C, James WP. A life course approach to diet, nutrition and the prevention of chronic diseases. Public Health Nutr 2004;7:101-21.

3. Sims EA, Horton ES. Endocrine and metabolic adaptation to obesity and starvation. Am J Clin Nutr 1968;21:1455-70.
4. Penke Z, Felszeghy K, Fernette B, et al. Postnatal maternal deprivation produces long-lasting modifications of the stress response, feeding and stress-related behaviour in the rat. Eur J Neurosci 2001;14:747-55.

5. Ioannidis JPA. Implausible results in human nutrition research. BMJ 2103;347:f6698. 